\title{
The Relationship between Stress and Conflict Handling Style in an ODR Environment
}

\author{
Paulo Novais, Davide Carneiro, Marco Gomes, and José Neves \\ CCTC/Department of Informatics, University of Minho, Braga, Portugal \\ \{pjon, dcarneiro, jneves\}@di.uminho.pt, \\ pg18373@alunos. uminho.pt
}

\begin{abstract}
Up until now, most approaches to Online Dispute Resolution focused on "traditional" problems such as the generation of solutions, the support to negotiation or the definition of strategies. Although these problems are evidently valid and important ones, research should also start to consider new potential issues that arise from technological evolution. In this paper we analyse the new challenges that emerge from resolving conflicts over telecommunications, namely in what concerns the lack of contextual information about parties. Specifically we build on a previous approach to stress estimation from the analysis of interaction and behavioural patterns. From the data gathered in a previous experiment we now trained classifiers that allow to assess stress in realtime, in a personalized and empirical way. With these classifiers, we were able to study how stress and conflict coping strategies evolve together. This paper briefly describes these classifiers, focusing afterwards on the results of the experiment.
\end{abstract}

Keywords: Online Dispute Resolution, Human-Computer Interaction, Behavioural Analysis, Negotiation.

\section{Introduction}

Unresolved or unmanaged conflicts often represent the largest reducible cost in an organization. Despite the extensive literature in social and organizational sciences that addresses conflicts, remarkable advances in computer sciences are scarce. Thus being, the current trend in Online Dispute Resolution (ODR) continues to focus mainly on the development of tools that can support the generation and exchange of proposals, document management and plain communication [4]. As a result, the actual ODR systems leave aside important issues that are present in traditional face-to-face dispute resolution processes, namely context information such as body language or behaviour [1]. Such issues have a preponderant role in human behaviour and communication [7]. As a consequence, the omission of this context information can influence the course of action and, consequently, the outcome in a conflict resolution scenario.

The use of Artificial Intelligence and, more particularly, Ambient Intelligence techniques can help suppress this gap [2]. This work aims to develop mechanisms that operate in a ODR-oriented virtual environment to collect context 
information and perceive the behavioural patterns of the users [5]. The approach followed consists in extending a simple and plain communication channel (e.g. instant messaging, e-mail, forums or even video-conferencing) with a context layer that can give meaning to what is said. This layer is pervasive and transparent. This is important since the consciousness of the monitoring may change the observed behaviour.

In the particular context of technology-supported conflict resolution, the ability to characterize a party's situation may be extremely relevant. A mediator may use such information to improve the odds of achieving successful outcomes by planning the right strategy, perceiving how each issue affects each party or detecting when it is time to take a break before emotions escalate. Similar activities are performed by human mediators in face-to-face settings. However, this is difficult to undertake in current ODR tools.

The proposed framework will provide valuable content to support the decision making process, overcoming some of the crucial needs of conflict resolution and management methods. One of the objectives is to abolish the use of the current self-reporting instruments and replace them with automated ones. Moreover these instruments must emphasize on perceiving the participants' behaviour in real time. This framework will monitor the conflict resolution process, tracking its dynamics and providing the decision maker with relevant information for taking well grounded decisions. It is integrated in the UMCourt conflict resolution platform, extending its services with a context layer.

\section{A Conflict Resolution Environment to Support Decision Making with Context Information}

The proposed framework builds on telecommunication technologies and on the UMCourt conflict resolution platform that provides a range of services previously developed such as a virtual negotiation environment, generation of ideas/solutions or information retrieval $10-12$.

On top of this base framework, a set of innovative functionalities were implemented to support the decision-making of the conflict manager by facilitating access to context information such as the conflict handling style of the parties or their levels of stress. This not only supports better decisions (by having access to more and relevant information) but also releases him for more complex issues such as the improvement of interpersonal communication and relationships.

The resulting environment thus encompasses several functionalities:

- Negotiation-oriented services - a set of supporting services were developed for the negotiation process, focusing on workflows, for the communication between the parties and for collecting and providing of information for the manager.

- Non-invasive estimation of stress - the main objective was to incorporate non-invasive methods for estimating the level of stress of the participants [13], 
which is of utmost importance in conflict resolution and can even be correlated with the conflict resolution style 14] and the level of escalation of the conflict. The effects of stress were successfully measured by analysing the interaction patterns of users with handheld devices, considering features such as touch intensity, acceleration of gestures, amount of movement or touch duration.

- Automatic estimation of personal conflict resolution styles - estimation of the conflict resolution style without the use of questionnaires [15] by using a generic model, with a theoretical background, using data from the workflows of the negotiation process in the context of conflicts.

Throughout the conflict resolution process, the main objective of the environment is to acquire data about the user that can be used to infer knowledge to characterize their behaviour. The variety of user types and the multitude of potential objectives of each particular environment demands an exhaustive analysis of all components to be included. Therefore, an intelligent environment with different sensors and devices was built, in order to provide several sources of information about the user's context and state (Figure 1). These devices are detailed in Table 1 and acquire different kinds of information from the user in a non-intrusive way concerning the way the user uses the interfaces or even the way the user moves.

While the user conscientiously interacts with the system and takes his/her decisions and actions, a parallel and transparent process takes place in which contextual and behavioural information is sent in a synchronized way to the conflict resolution platform. The platform, upon converting the sensory information into useful knowledge, allows for a contextualized analysis of the user's behaviour. This contextualized analysis may be performed by the platform itself (e.g. for performing decision-support related tasks such as classification of the current conflict handling style) or by the mediator. When the parties receive feedback from the platform (e.g. a new proposal), it may also include some kind of feedback from their state (e.g. an avatar depicting the level of stress/escalation of the other participants). This will allow every participant to take better framed decisions.

Table 1. Brief description of the functionalities of the devices that constitute the environment

\begin{tabular}{|l|l|l|}
\hline Device & Brief Description & Main features \\
\hline \hline HP Touchsmart & All-in-one PC & touchscreen, web cam, large screen \\
\hline Samsung Galaxy Tab & Tablet PC & $\begin{array}{l}\text { touchscreen, web cam, accelerome- } \\
\text { ter, relatively large screen, mobile, } \\
\text { Android OS }\end{array}$ \\
\hline HTC PDAs & Smartphones & $\begin{array}{l}\text { touchscreen, camera, accelerome- } \\
\text { ter, mobile, Android OS }\end{array}$ \\
\hline
\end{tabular}




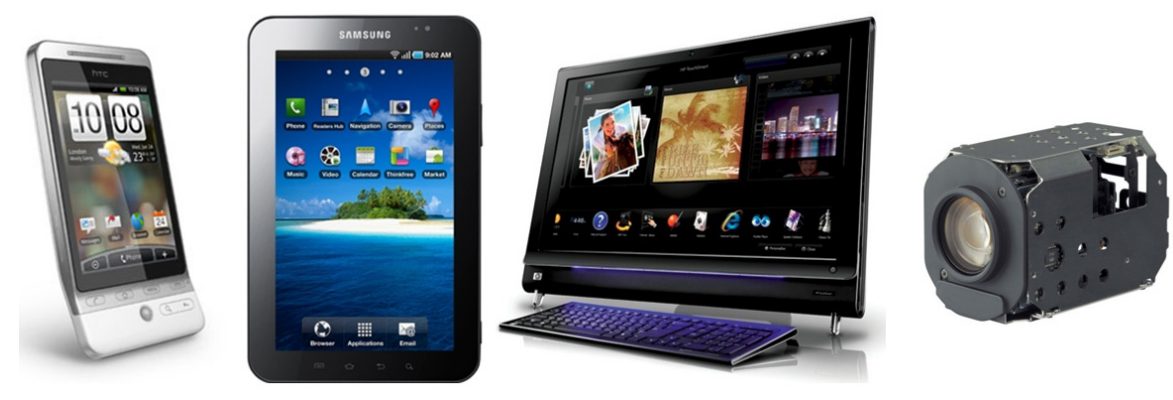

Fig. 1. The devices that make up the environment

\subsection{Feature Extraction}

Extraction of representative features from the sensor data provided by the described devices is paramount for the construction of the high-level information model. The studied features are extracted from multiple sensor observations, and combined into a single concatenated feature vector which generates or triggers system actions based on template methods. The features studied can be organized as follows:

Context Features. The context of the user, in all its different dimensions (e.g. socio-economical, geographical, historical, personal), constitutes significant information that can allow the remaining participants to correctly interpret his decisions and actions. Context features will thus characterize the activity of each party within the conflict resolution platform. Some of these features can be studied through questionnaires or by profiling techniques while others can be studied in a non-intrusive way, by analysing the nature of the actions performed. As an example, the number and utility of the proposals generated by a given party, duly framed in the conflict's specificities, may allow to infer the objectives of the party (e.g. maximization of personal gain).

Physical Features. These features describe the behaviour of the participant's bodies and can be co-related with aspects such as the level of stress, escalation or excitement. Particularly, we are interested in studying how a user moves within its environment, how fast, and in which ways. In order to acquire information for these features we are using image processing techniques that analyse video feeds from the user's environment. Particularly, we focus on the estimation of the amount of movement over time, which we have previously determined to be related to the level of stress [16]. The image processing stack uses the principles established by [17] and image differentiation techniques to calculate the amount of movement of a user between two consecutive frames [18]. 
Behavioural Features. Our behaviour influences many aspects of our daily interactions with our surroundings. In this work we are focusing on three particular features that can be studied in a non-intrusive way.

- touch patterns - the touch pattern represents the way in which a user touches a given device. In a few words it describes a variation of intensity over a period of time. Each user has a particular touch pattern, with a specific intensity curve, that can be studied by fitting a quadratic curve. This information is acquired from touch screens with support for touch intensity.

- touch intensity - the intensity of the touch represents the amount of force that the user is putting into the touch in a given moment. It is analysed in terms of the maximum, minimum and mean intensity of each touch event. This information is acquired from touch screens.

- agitation level - the level of agitation of the user is given in terms of the nature of their movements. This information is extracted from the video feeds as well as from accelerometers placed on handheld objects. Particularly interesting are the accelerometers of Android platforms, which are easily accessible.

Performance Features. The performance features relate to the accuracy and efficacy with which a user performs given tasks. Two features are studied in detail in our approach:

- touch accuracy - a measure of touches or clicks in active controls versus touches in passive areas (e.g. without controls, empty areas) in which there is no sense in touching. This feature is strictly related to aspects such as stress or fatigue [16]. This information is acquired from the several devices on the environment with which the user may interact.

- response - this feature represents the time span between the beginning and the end of the user's responses to the process interactions. This data is acquired from the different devices with which the user can interact.

\section{Inference Process}

To integrate the multimodal features studied in this work, a decision level integration strategy was used. The decision level fusion methods used include weighted decision methods and machine learning techniques. This section describes the data level integration of the contextual and behavioural features acquired from the available devices in the environment. We focus on the generation of knowledge describing two high level concepts: personal conflict handling styles and stress.

The classification of the personal conflict handling style of an individual is traditionally performed in psychology through the use of questionnaires [9]. This method, although well studied and established, has as main disadvantages the fact that it is static (does not adapt to changes in the individual's state), that it is easy to lie in a questionnaire and that it is not straightforward to develop 
questionnaires that can accurately characterize the intended reality. One of the main advantages of the approach implemented is that it does not use these selfreporting instruments. It rather relies on the analysis of the proposals exchanged in terms of their utility framed in the context of each case. Five conflict handling styles are considered in this work, as defined by [6].

Boundary utility values (e.g. BATNA, WATNA) [8] are determined using predetermined information specific for each case. The personal handling conflict style is classified using data extracted from the proposals' workflow by determining how close the utility of the proposals are to boundary or central values (Figure 2). The whole process of extracting information about the conflict handling style from consecutive proposals is described in detail in [15].

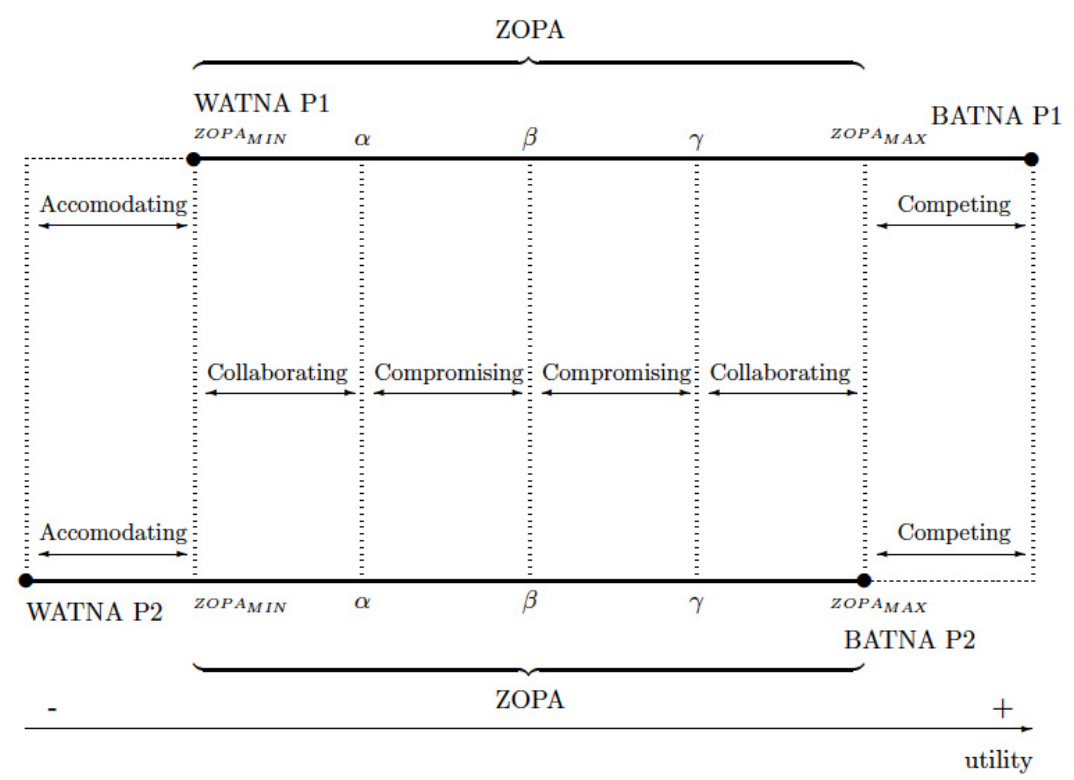

Fig. 2. The space that defines the personal conflict style in terms of the distance to specific points of the zone of possible agreement

When the conflict resolution system has a temporal representation of the evolution of the conflict styles, it may implement dynamic conflict resolution methods that adapt strategies in real time. This can also be done by the mediator whom, by using such approaches, gains a complementary view to the legal aspects of the decision-making processes.

Concerning the classification of the level of stress, the main objective is to apply machine-learning algorithms that can classify data from some of the features studied that are related to stress. The experiments performed allowed to determine how increased levels of stress affect given aspects of such features [16]. 
With the data collected in that experiment we now trained classifiers that allow to assess the level of stress of the users, in real time, taking as input the features described in section 2.1. A brief analysis of these new classifiers, that constitute the backbone of this study, is performed in section 5.1

\section{Analysing Behavioural Patterns in Real-Time in a Negotiation}

In order to achieve the results documented in this paper a test environment was set up in the Intelligent Systems Lab of the University of Minhd1. In this environment, the users are isolated from external stimuli and play a negotiation game that implies the interaction with the devices. The collection of the data was organized into two phases. In a first phase, test subjects were required to perform these tasks in a stress-free environment. In a second phase, the users performed the same tasks subject to stressors such as the vibration of the devices, loud and annoying sounds, unexpected behaviours of the devices, among others.

\subsection{The Negotiation Game}

The negotiation game simulates a business situation in which each party must achieve a desired outcome in the negotiation or go bankrupt. The optimal result is a win/win situation for both parties. The game starts with the application randomly giving one of the predetermined roles to each party. The instructions to win the game were to negotiate a successful deal and make sure that the party in question didn't go bankrupt. Each party's instructions were clearly presented, visible to them through the application interfaces. The objectives and the persona for each party are given:

- Role A - party A is a light bulb manufacturer who specializes in specific types of light bulbs. He is however not the only supplier of this light bulb. In order to stay in business, he needs to sell 6,000 light bulbs at 1 euro or more per light bulb. If he does not achieve this, he goes bankrupt. Party A is also given the information that Party $\mathrm{B}$ also needs to make this deal.

- Role B - party B is a retailer of light bulbs. He recently signed a contract to supply a hotel chain 6,000 of these specific light bulbs. The hotel is prepared to pay 2 euros per light bulb. If Party B does not manage to negotiate with Party A to buy the light bulbs at 1,20 or less, he will go bankrupt. Party B is told that party $\mathrm{A}$ is in a financial trouble and needs to make the deal to survive.

The game is to last at most ten rounds (in the training phase) or five minutes (in the stressful phase). If a successful outcome is not achieved in this time, both parties go bankrupt. The ZOPA (Zone of Potential Agreement) [22] is bounded by the BATNA ( 1 euro) and the WATNA (1,20 euro). The range of possible agreement is 0.20 , but the parties are not aware of this detail.

\footnotetext{
${ }^{1}$ The website of the Intelligent System Lab is available at http://islab.di. uminho.pt (accessed in August, 2012).
} 


\subsection{Statistical Data Analysis}

While the parties play this game, data about their behaviour is collected in order to be analysed. To determine to which extent each feature that was considered is or is not influenced by stress for each user, data from both phases is compared. Provided that most of the distributions are not normal, the Mann-Whitney test is used to perform the analysis. This test is a nonparametric statistical hypothesis test for assessing whether one of two samples of independent observations tends to have larger values than the other.

The null hypothesis is thus: $H_{0}=$ The medians of the two distributions are equal. For each two distributions compared, the test returns a $p$-value, with a small $p$-value suggesting that it is unlikely that $H_{0}$ is true. For each parameter, data from both phases is compared. In all the tests, a value of $\alpha=0.05$ is used.

Thus, for every Mann-Whitney test whose $p$-value $<\alpha$, the difference is considered to be statistically significant, i.e., $H_{0}$ is rejected. A significant difference between data from the two phases means that the parameter is effectively influenced by stress for this specific user. Table 2 describes the dataset used in this experiment.

Table 2. Summary of the data generated during the experiment. The size of the datasets comprises all the data generated, i.e., first and second phase.

\begin{tabular}{|l|l|l|}
\hline Data & Brief Description & Size \\
\hline \hline Acceleration & $\begin{array}{l}\text { Data concerning the acceleration } \\
\text { felt on the handheld device while } \\
\text { playing the game }\end{array}$ & 3366 \\
\hline Movement & $\begin{array}{l}\text { A dataset containing information } \\
\text { about the amount of movement } \\
\text { during the tests }\end{array}$ & 9137 \\
\hline Touches & $\begin{array}{l}\text { This dataset contains information } \\
\text { about the touches }\end{array}$ & 590 \\
\hline Proposals & $\begin{array}{l}\text { Data concerning the proposals } \\
\text { made by the parties in all rounds }\end{array}$ & 60 \\
\hline
\end{tabular}

\section{$5 \quad$ Experiment Results}

Stress is a highly subjective phenomenon, with each individual responding differently to the same stressors [23]. In that sense there is the need to develop personalized models that can adequately shape the individual's response to stressors. In the first subsection that follows, we detail the process of developing personalized stress models that can be used in real-time. In the second one, we detail the results of using this approach in conjunction with the assessment of the conflict handling style, allowing us to understand how individuals cope with conflict under stress (or under the lack of it). 


\subsection{Empirical Measurement of Stress}

In order to develop personalized stress models, in a previous experiment we collected data about how each user behaves within an environment and how he interacts with the smartphone. The experiment consisted in collecting data describing these variables with the users under and without the effect of stressors. The statistical analysis of the data allowed to build personalized stress response models. The features considered and the dataset built are described in Table 3. The whole process of data collection and analysis is described in more detail in [16].

Table 3. Summary of the data generated during the stress experiments

\begin{tabular}{|l|l|l|}
\hline data & Brief Description & Size \\
\hline \hline Acceleration & $\begin{array}{l}\text { Data concerning the acceleration felt on } \\
\text { the handheld device while playing the } \\
\text { game }\end{array}$ & 27291 \\
\hline Maximum intensity of touch & $\begin{array}{l}\text { Data about the maximum intensity of each } \\
\text { touch in a touchscreen }\end{array}$ & 1825 \\
\hline Mean intensity of touch & $\begin{array}{l}\text { This dataset contains data about the mean } \\
\text { intensity of each touch event in a touch- } \\
\text { screen }\end{array}$ & 1825 \\
\hline Amount of movement & $\begin{array}{l}\text { A dataset containing information about } \\
\text { the amount of movement during tests }\end{array}$ & 25416 \\
\hline Touches on target & $\begin{array}{l}\text { This dataset contains information about } \\
\text { the accuracy of the touches }\end{array}$ & 1825 \\
\hline Stressed touches & $\begin{array}{l}\text { A dataset containing information that al- } \\
\text { lows to classify each touch as stressed or } \\
\text { not stressed }\end{array}$ & 1825 \\
\hline Score & $\begin{array}{l}\text { A dataset describing the performance of } \\
\text { the user playing the game, during the tests }\end{array}$ & 321 \\
\hline Touch duration & $\begin{array}{l}\text { A dataset containing the duration of each } \\
\text { touch event }\end{array}$ & 1825 \\
\hline
\end{tabular}

In recent work, we used these datasets describing how each user reacts before stress, to train personalized classifiers: one for each feature and each user. Our approach consisted in using a standard and well known pattern recognition tool: the $k$-nearest neighbour algorithm. Specifically, we are using the weka.classifiers.lazy.IBk [24] implementation for java, using the Weka workbench (Weka 3.6.3) [21]. It is a method for classifying objects based on closest training examples in the feature space: an object is classified by a majority vote of its neighbours, with the object being assigned to the class most common amongst its $\mathrm{k}$ nearest neighbours ( $\mathrm{k}$ is a positive integer, typically small). If $\mathrm{k}=$ 1 , then the object is simply assigned to the class of its nearest neighbour.

Figure 3 depicts the working of this algorithm with a real example. The data detailed describes several instances of the maximum intensity of touch: squares 
correspond to the data collected under stress while circles correspond to the data collected without stress. This data shows the same tendency depicted above: under stress the intensity of touch is higher. Given the new instance to be classified, represented in this case by a star symbol (a new touch), and a $\mathrm{k}=4$, the algorithm would classify it as stressed given that there are more neighbours from the class "Stressed" (3) than from the class "not stressed" (1). The working of the algorithm is the same for each of the other parameters and for each new instance that must be classified.

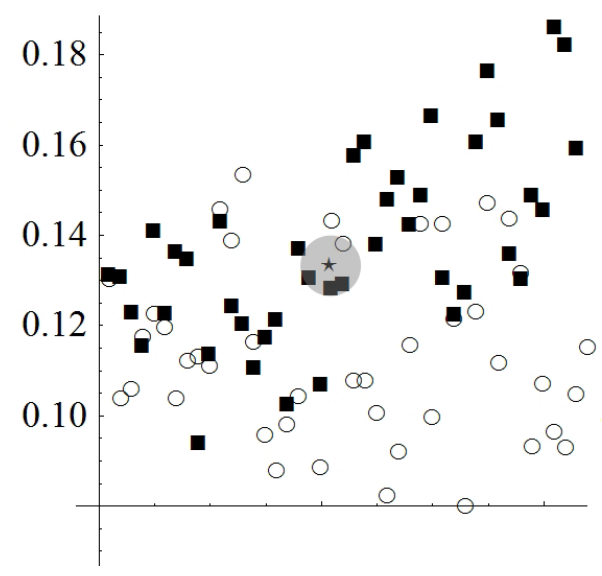

Fig. 3. Example of the working of the nearest neighbour algorithm by a majority vote: in this case the star symbol (representing the new instance) would be classified as "stressed" (the class represented by squares)

The conclusions achieved when analysing the performance of the classifiers are briefly summarized in table 4. In each row the table describes the name of the parameter as well as the the best and worst classifier trained for all the users and the respective kappa coefficients. Finally, for each parameter it also shows the average performance of the classifiers, which is a more significant indicator of how fit each feature is for measuring stress.

Table 4. Summary of the results of the analysis of the performance of the classifiers

\begin{tabular}{|l|c|c|c|c|c|c|}
\hline \multirow{2}{*}{ Dataset } & \multicolumn{2}{|c|}{ Best } & \multicolumn{2}{c|}{ Worst } & \multicolumn{2}{c|}{ Average } \\
\cline { 2 - 7 } & $\%$ & $\kappa$ & $\%$ & $\kappa$ & $\%$ & $\bar{\kappa}$ \\
\hline \hline Acceleration. & 99.85 & 0.995 & 95.36 & 0.866 & 98.1 & 0.94 \\
\hline Amount of Mov. & 97.41 & 0.86 & 56.15 & 0.03 & 78.84 & 0.23 \\
\hline Max. Touch Intensity & 86.46 & 0.71 & 71.82 & 0.21 & 77.56 & 0.43 \\
\hline Avg. Touch Intensity & 100 & 1.0 & 87.79 & 0.69 & 95.13 & 0.89 \\
\hline Touch Duration & 93.92 & 0.86 & 80.37 & 0.56 & 87.32 & 0.71 \\
\hline
\end{tabular}


Using the classifiers trained it is possible to build a solution for estimating the level of stress of users, in a personalized way and in real time, since each user has a number of classifiers that were trained using his personal interaction patterns. These can thus be used in real time to classify each instance of data being generated by the sensors. A mediator can thus access the data concerning the level of stress of each party or build a representation of the average level of stress of the conflict resolution environment, allowing him to intervene if emotions escalate. This is done with the aim of preserving human relationships and bonds, which are crucial for a successful outcome. This real-time solution has been used in the Negotiation game implemented in this experiment, which allowed to correlate the level of stress with conflict coping strategies. The results of this study are detailed in the following subsection.

\subsection{Stress and Conflict Handling Styles}

In this subsection we make an analysis of the effect of stressors on the conflict handling style of the parties. In order to do it we compared the data of the two phases of the game (without and with stressors) for the same pairs of players in search for statistically significant differences due to the action of the stressors. The main aim of the study is to assess the influence of stress on the behaviour of the parties and on the outcome of the negotiated process. This experiment involved 14 users playing the game, in a total of 60 negotiation rounds. The data gathered included inputs from the devices, which was used to estimate the level of stress of each user using the models developed in previous work [16]. These models were used to determine how the level of stress relates with the behaviour of the participants in a negotiation.

In order to statistically deal with this data, a numeric scale was used to describe the conflict handling styles. Table 5 depicts the conflict handling styles considered, the number of times that each style was evidenced by each participant and the ordinal rank attributed in order to be used by data-mining algorithms. The exact numeric quantity of a particular value has no significance beyond its ability to establish a ranking over a set of data points. Therefore, rank-ordering was used which describes an order but does not establish relative size or degree of difference between the items measured. This was a mandatory step to make the data suitable for statistical and machine-learning techniques.

One of the first conclusions achieved when analysing the data is that parties show a competitive style of negotiation most of the times, both in stressed and calm settings. However, when calm, the use of more cooperative style is slightly larger. The histograms depicted in figure 4 depict this: the dashed curve refers to the distribution of the conflict handling styles in the calm phase while the solid line refers to the distribution in the stressed phase. However, the differences are not statistically significant (MannWhitneyTest $=0.33$ ). On average, the style of a party when stressed is 1.525 (closer to competing) and when calm is 1.74 (closer to collaborating).

The evaluation of the progress of the conflict styles during the negotiation process was centred on the average slope of its numeric values. In other words, 
Table 5. Summary of the conflict handling styles, the number of times that each style was evidenced and the rank-ordering of each style

\begin{tabular}{|l|l|l|}
\hline Conflict Handling Style & $\begin{array}{l}\text { Number of times used during the } \\
\text { game }\end{array}$ & Ordinal rank \\
\hline \hline Competing & 30 & 1 \\
\hline Collaborating & 12 & 2 \\
\hline Compromising & 8 & 3 \\
\hline Accommodating & 10 & 4 \\
\hline Avoiding & 0 & 5 \\
\hline
\end{tabular}

the object of study was the variation of the conflict styles used by each party within the negotiation game. It was concluded that in a stressful state the parties tend to vary their conflict handling style more (on average 0.71 points between the beginning and the end of the game) than when they are calm (on average 0.61 points). This is in line with other results that point out to more sudden and less weighted decisions under stress. Besides that, it was also concluded that the 'manufacturer' role presents a higher average slope (faster change in the conflict style), being on average 0.83 than the 'retailer' (on average 0.51 points).

Let us now analyse the values of the proposals exchanged by the parties during the negotiation. It can be concluded that both parties change more the values of the proposals (on average 0.19) when under stress than during the calm phase (on average 0.14 ). Moreover, the 'manufacturers' present a more dynamic proposal evolution (changing 0.19 in average) than the 'retailers' (average slope is 0.11).

The euclidean distance to the optimum value was also analysed, i.e., it was studied the deviation given the most desirable negotiation outcome (the value that prevented both parties from entering into bankruptcy). Under a stressful situation both parties were at a distance of, in average, 0.154 euros from the optimum value while in stress-free situation the distance decreases to 0.071 euros, in average. Therefore, it can be concluded that in a stressful situation it is more likely that the parties propose more uncooperative values. This can be explained as a consequence of acting too quickly or relying too much on coercion. When parties are under pressure they can commit strategic mistakes or give in unwanted concessions. It may also lead to bad agreements.

Acting too quickly is also a known response to external and internal stressors. Indeed, considering the duration of the rounds, one can state that $90 \%$ of the negotiation rounds had a shorter duration under a stressful environment than under a stress-free one. However, only in $30 \%$ of these cases was the different statistically significant (at a level of 0.05).

Concerning the evolution of the conflict handling style in each game played, it is possible to conclude that $80 \%$ of the participants used a competitive conflict style, which is assertive and uncooperative, in the early rounds. During the game $55 \%$ of the players improve their styles (shifting towards more cooperative solutions), $35 \%$ remain on the same style and $10 \%$ become more competitive. It is stated that 'competitors' often use power as the primary tool for handling 


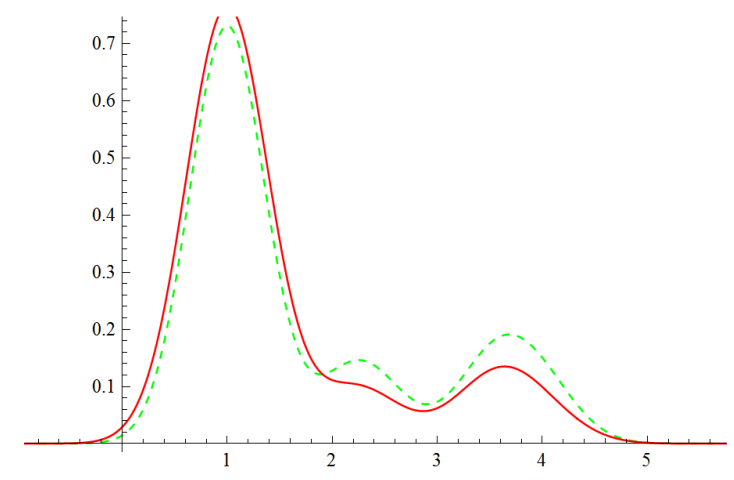

Fig. 4. Distribution of the styles used by the parties: the normal line represents data from the stressed phase while the dashed line represents data from the calm phase. The $\mathrm{X}$ axis represents the ordinal rank of the conflict handling styles as defined in Table 5 In a calm state the users evidence more cooperative styles.

conflict, and work to prove the importance of one side of the argument in order to win. This can be one explanation. Otherwise, they are usually more concerned with winning the game than finding the best solution. Taking into consideration the pre-conditions of the game, the second hypothesis is more plausible.

In order to provide a more specific view of the results, one actual case is highlighted. It shows the evolution of the values proposed during the negotiation with stress (Figure 5 (a)) and without stress (Figure 5 (b)). The normal line represents the values proposed by the 'retailer' and the dashed one by the 'manufacturer'. It is possible to see that the 'manufacturer' is more flexible (changes more often). This is a recurrent behaviour and can be explained by the fact that the seller (in this case, the 'manufacturer' role), in a buyer's market, needs to be more flexible and expect more negotiation about contingencies. Comparing the lines, in a calm state the 'manufacturer's' average slope is 0.31 and the 'retailer's' is 0.056 . When under stress, the values rise to 0.5 and 0.1 , respectively. Similar results are also observed in other pairs of players and are in line with the previously described conclusions: stressed participants take hastier and less weighted decisions.

\section{Conclusions and Future Work}

In a general way, the results achieved were consistent for the majority of the participants. They can be summarized as follows:

- Stressed participants take hastier decisions, taking less time to think them through;

- Stressed participants are more prone to change their behaviour and do it in more significant ways (we focused on the values of the proposals exchanged and on the conflict resolution style evidenced); 


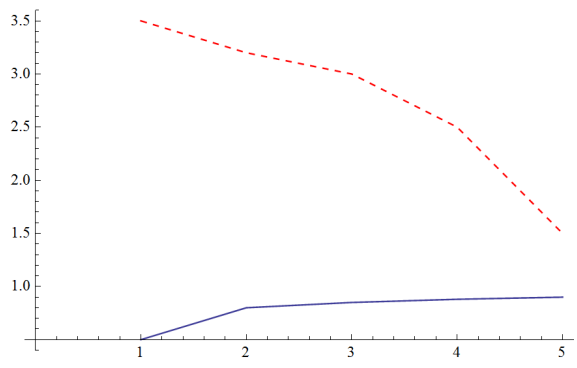

(a)

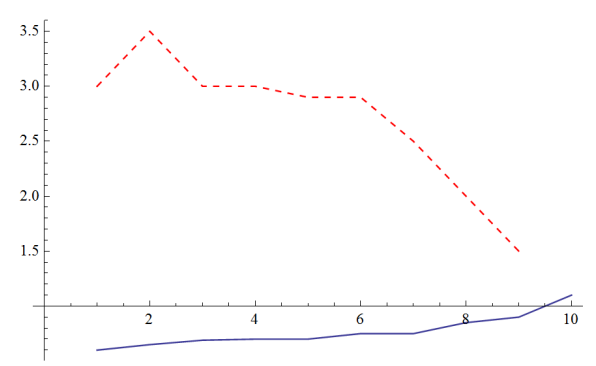

(b)

Fig. 5. Evolution of the values proposed during the negotiation when under stress (a) and without stress (b). The dashed line depicts the values proposed by the manufacturer while the normal line depicts the values proposed by the retailer. It is possible the see that under stress the values proposed vary faster.

- Under a stressful environment, outcomes tend to be farther away from the optimum result;

- Under stress participants tend to be more competitive;

These results stress the need for seeking calm and harmonious environments for conflict resolution. As a consequence, it can be seen once more that courtrooms are not the ideal conflict resolution environment as these are highly competitive milieus in which parties forget each other's natural ambitions and focus on the maximization of the own gain. Alternative environments, focused on cooperative strategies, should be preferred.

Moreover, in order for conflict managers to improve their action, access to the context information mentioned should be provided. This would allow them to detect, in due time, an escalation on the level of conflict and prevent a degradation on the relationships. This context information, that is available in face-to-face settings, must also be considered in virtual settings so that conflict managers can increase the efficiency of their decisions by considering more complete information.

Despite its apparent advantages, this approach may also encompass risks. One of the main concerns raised is related with the risk of people trying to control the system if they know how it works. On the one hand it is known that people have tried to cheat systems as much as they try to make them cheat-proof (e.g. parties in court will also try to manipulate decision-makers leading them into believing what they want). We argue that in this approach this kind of behaviours may be hindered. On the one hand, the process is transparent, i.e., parties will not be aware of how the process of compiling this information is implemented. On the other hand, even if parties try to cheat the system, it is difficult to fake expressions, gestures or other behavioural features since they are more reflexes than conscious behaviours. 
Given this, we conclude that this approach may encompass several interesting advantages for mediators, specifically for the ones operating in online environments, allowing them to take more informed decisions. Work will continue by including additional sources of information, such as mouse and keyboard interaction patterns. The main aim is to have a multitude of inputs that enable the construction of a seamless environment for the acquisition of context information, based on several different devices, as rich as possible.

Acknowledgments. This work is funded by National Funds through the FCT - Fundação para a Ciência e a Tecnologia (Portuguese Foundation for Science and Technology) within projects PEst-OE/EEI/UI0752/2011 and PTDC/EEISII/1386/2012. The work of Davide Carneiro is also supported by a doctoral grant by FCT (SFRH/BD/64890/2009).

\section{References}

1. James, W.: What is an Emotion? Mind 9(34), 188-205 (1884)

2. Lodder, A., Thiessen, E.: The role of artificial intelligence in online dispute resolution. In: Workshop on Online Dispute Resolution at the International Conference on Artificial Intelligence and Law, Edinburgh, UK (2003)

3. Healey, J., Picard, R.W.: Detecting stress during realworld driving tasks using physiological sensors. IEEE Transactions on Intelligent Transportation Systems 6(2), 156-166 (2005)

4. Katsch, E., Rifkin, J.: Online dispute resolution - resolving conflicts in cyberspace. Jossey-Bass Wiley Company, San Francisco (2001)

5. Bharat, A., Solomon, J.S.: The effect of task complexity and conflict handling styles on computer-supported negotiations. Information and Management 37(4), 161-168 (2000)

6. Kilmann, R.H., Thomas, K.W.: Developing a Forced-Choice Measure of ConflictHandling Behavior: The MODE Instrument. Educational and Psychological Measurement (1977)

7. Liao, W., Zhang, W., Zhu, Z., Ji, Q., Gray, W.: Toward a decision-theoretic framework for affect recognition and user assistance. International Journal of HumanComputer Studies 64(9), 847-873 (2006)

8. Notini, J.: Effective alternatives analysis in mediation: "batna/watna" analysis demystified (2005) (last accessed August 2012)

9. Rahim, M.A.: A measure of styles of handling interpersonal conflict. The Academy of Management Journal 26(2), 368-376 (1983)

10. Andrade, F., Novais, P., Carneiro, D., Zeleznikow, J., Neves, J.: Using BATNAs and WATNAs in Online Dispute Resolution. In: Nakakoji, K., Murakami, Y., McCready, E. (eds.) JSAI-isAI 2009. LNCS (LNAI), vol. 6284, pp. 5-18. Springer, Heidelberg (2010)

11. Carneiro, D., Novais, P., Andrade, F., Zeleznikow, J., Neves, J.: Using Case-based Reasoning to Support Alternative Dispute Resolution. In: de Leon F. de Carvalho, A.P., Rodríguez-González, S., De Paz Santana, J.F., Rodríguez, J.M.C. (eds.) Distributed Computing and Artificial Intelligence. AISC, vol. 79, pp. 123-130. Springer, Heidelberg (2010) 
12. Carneiro, D., Novais, P., Andrade, F., Neves, J.: Retrieving Information in Online Dispute Resolution Platforms: A Hybrid Method. In: Proceedings of the Thirteenth International Conference on Artificial Intelligence and Law, University of Pittsburgh School of Law. ACM (2011) ISBN: 978-1-4503-0755-0

13. Gomes, M., Carneiro, D., Novais, P., Neves, J.: Modelling stress recognition in conflict resolution scenarios. In: Corchado, E., Snášel, V., Abraham, A., Woźniak, M., Graña, M., Cho, S.-B. (eds.) HAIS 2012, Part I. LNCS, vol. 7208, pp. 533-544. Springer, Heidelberg (2012)

14. Simon, T., Friedman, R.: Conflict style and coping with role conflict: An extension of the uncertainty model of work stress. International Journal of Conflict Management 13(3), 236-257 (2002)

15. Carneiro, D., Gomes, M., Novais, P., Neves, J.: Developing dynamic conflict resolution models based on the interpretation of personal conflict styles. In: Antunes, L., Pinto, H.S. (eds.) EPIA 2011. LNCS, vol. 7026, pp. 44-58. Springer, Heidelberg (2011)

16. Carneiro, D., Castillo, J.C., Novais, P., Fernández-Caballero, A., Neves, J.: Multimodal Behavioural Analysis for Non-invasive Stress Detection. Expert Systems with Applications 39(18), 13376-13389 (2012),

http://dx.doi.org/10.1016/j.eswa.2012.05.065

17. Castillo, J.C., Rivas-Casado, A., Fernández-Caballero, A., López, M.T., MartínezTomás, R.: A multisensory monitoring and interpretation framework based on the model-view-controller paradigm. In: Ferrández, J.M., Álvarez Sánchez, J.R., de la Paz, F., Toledo, F.J. (eds.) IWINAC 2011, Part I. LNCS, vol. 6686, pp. 441-450. Springer, Heidelberg (2011)

18. Fernández-Caballero, A., Castillo, J.C., Martínez-Cantos, J., Martínez-Tomás, R.: Optical flow or image subtraction in human detection from infrared camera on mobile robot. Robotics and Autonomous Systems 58(12), 1273-1281 (2010)

19. Quinlan, R.: C4.5: Programs for Machine Learning. Morgan Kaufmann Publishers, San Mateo (1993)

20. Platt, J.: Fast Training of Support Vector Machines using Sequential Minimal Optimization. In: Schoelkopf, B., Burges, C., Smola, A. (eds.) Advances in Kernel Methods - Support Vector Learning (1998)

21. Holmes, G., Donkin, A., Witten, I.H.: Weka: A machine learning workbench. In: Proc. Second Australia and New Zealand Conference on Intelligent Information Systems, Brisbane, Australia (1994)

22. Raiffa, H.: Art and Science of Negotiation. Harvard University Press (1982)

23. Selye, H.: The Stress of Life. McGraw-Hill (1978)

24. Aha, D., Kibler, D.: Instance-based learning algorithms. Machine Learning 6, 37-66 (1991) 